\title{
CELLULAR STRINGS ON POLYTOPES
}

\author{
L. J. BILLERA, M. M. KAPRANOV, AND B. STURMFELS
}

(Communicated by Jeffry N. Kahn)

\begin{abstract}
The complex of cellular strings with respect to a generic linear functional on a $d$-dimensional convex polytope has the homotopy type of the $(d-2)$-sphere. This result was conjectured in a special case by H.-J. Baues.
\end{abstract}

\section{INTRODUCTION}

While from a topological point of view all convex polytopes have the same boundary, the rich combinatorial structure of these objects has been studied since the earliest days of mathematics. This paper deals with a new combinatorial invariant, the poset of cellular strings on a polytope. We determine the homotopy type of this poset, and as a corollary we prove a conjecture of Baues in the theory of loop spaces.

Baues's conjecture arose from the problem of finding explicit CW-models for iterated loop spaces $\Omega^{i} X$ of a given CW-space $X$. Using cellular strings on cubes, Baues [2] demonstrated that the algebraic cobar construction of Adams [1] can be strengthened to a geometric model for $\Omega X$. More precisely, if $X$ is a simplicial space [6] with one vertex and no edges, then Baues's model $\underline{\Omega} X$ is a space glued from cubes, where each cube $I^{n-1}$ approximates the space of paths in the simplex $\Delta_{n}$ joining the 0 th and the $n$th vertex. Here faces of $I^{n-1}$ correspond to cellular strings on $\Delta_{n}$.

Milgram [8] noted that the $(n-1)$-dimensional permutohedron $P_{n}$ (the convex hull of a generic orbit of the symmetric group $S_{n}$ in $\mathbf{R}^{n}$ ) similarly approximates the path space of the cube $I^{n}$. More precisely, the face poset of $P_{n}$ is isomorphic to the poset of cellular strings in $I^{n}$. This permitted Baues to iterate his geometric cobar-construction and apply it to cubical spaces $X$ with trivial 1-skeleton. He constructed a CW-space $\underline{\Omega} X$, glued from products of permutohedra, which is a model for $\Omega X$. In particular, if $Y$ is a simplicial space with trivial 2-skeleton and $X=\underline{\Omega} Y$, then $\underline{\Omega} X$ is a model for the iterated loop space $\Omega^{2} Y$.

One is tempted to continue this process, introducing "hyperpermutohedra" as complexes of cellular strings in $P_{n}$ and so on. However, these complexes are

Received by the editors February 1, 1993.

1991 Mathematics Subject Classification. Primary 52B11; Secondary 55P35.

Partially supported by ARO (DAAG29-85-C-0018) and NSF (DMS-9004123, DMS-9002056) at Cornell University. This work was done while the second author was visiting the Mathematical Sciences Institute at Cornell. 
no longer isomorphic to face complexes of convex polytopes (and even fail to be spheres). Nevertheless, Baues conjectured that they are homotopy equivalent to spheres and that one can choose a subcomplex homeomorphic to a sphere whose embedding is a homotopy equivalence [2, Conjecture 7.4].

In $\S 1$ we prove Baues's conjecture for an arbitrary convex polytope $P \subset \mathbf{R}^{n}$ whose vertices are ordered by means of a generic linear functional $f$. In $\S 2$ we show that the desired subcomplex homeomorphic to a sphere can be taken to be the boundary complex of the monotone path polytope $\Sigma_{f}(P)$. These results open up the possibility of iterating Baues's construction to get CW-models for iterated loop spaces by gluing products of iterated monotone path polytopes. In $\S 3$ we discuss a natural generalization of Baues's conjecture arising from the theory of fiber polytopes [3].

We are grateful to David Stone for bringing the work of Adams and Baues to our attention and to Anders Björner and Günter Ziegler for helpful discussions.

\section{SPHERICITY OF THE CELlulaR STRING COMPLEX}

Let $P \subset \mathbf{R}^{n}$ be an $n$-dimensional convex polytope. A stippling on $P$ (see [2, Definition III.2.3]) is a rule which assigns to each face $\Gamma$ of $P$ a vertex $\eta(\Gamma)$ of $\Gamma$ such that whenever $\Gamma_{2}$ is a face of $\Gamma_{1}$ and $\eta\left(\Gamma_{1}\right) \in \Gamma_{2}$, then $\eta\left(\Gamma_{1}\right)=\eta\left(\Gamma_{2}\right)$.

An important class of stipplings is provided by convex geometry. Let $f$ : $\mathbf{R}^{n} \rightarrow \mathbf{R}$ be a linear functional. We say that $f$ is generic with respect to $P$ if it is nonconstant on each edge of $P$. In this case on each face $\Gamma \subset P$ the function $f$ attains its minimum (resp. maximum) in exactly one point, which is a vertex denoted $s_{f}(\Gamma)$ (resp. $\left.t_{f}(\Gamma)\right)$.

Proposition 1.1. If $f$ is a generic linear functional on $P$, then $s_{f}$ and $t_{f}$ are stipplings.

Let $P$ be a polytope with a fixed pair of stipplings $(s, t)$. A cellular string on $P$ is a sequence of proper faces $\left(\Gamma_{0}, \Gamma_{1}, \ldots, \Gamma_{m}\right)$ such that $s\left(\Gamma_{0}\right)=$ $s(P), t\left(\Gamma_{m}\right)=t(P)$, and $s\left(\Gamma_{i}\right)=t\left(\Gamma_{i-1}\right)$ for each $i=1,2, \ldots, m$. The set $\omega=\omega(P, s, t)$ of all cellular strings is partially ordered by the relation $\left(\Gamma_{0}, \Gamma_{1}, \ldots, \Gamma_{m}\right) \leq\left(\Delta_{0}, \Delta_{1}, \ldots, \Delta_{k}\right)$ if there exist $0=i_{0}<i_{1}<i_{2}<\cdots<$ $i_{k}<i_{k+1}=m$ such that $\left(\Gamma_{i_{\nu}}, \Gamma_{i_{\nu}+1}, \ldots, \Gamma_{i_{\nu+1}}\right)$ is a cellular string on the face $\Delta_{\nu}$, for each $\nu=0,1, \ldots, k$.

The geometric realization $|\omega|$ of the simplicial complex of chains in $\omega$ can be viewed as a model for the space of all monotone paths on the boundary $\partial P$ joining $s(P)$ and $t(P)$. So it is a natural question whether $|\omega|$ has the homotopy type of the $(n-2)$-sphere $S^{n-2}$. The following affirmative answer is the main result of this paper.

Theorem 1.2. Let $f$ be a generic linear functional on an $n$-dimensional convex polytope $P \subset \mathbf{R}^{n}$. Then the poset $\omega\left(P, s_{f}, t_{f}\right)$ is homotopy equivalent to $S^{n-2}$.

Proof. Let $\lambda_{1}<\lambda_{2}<\cdots<\lambda_{N}$ be the values of $f$ at the vertices of $P$, so the segment $Q:=\left[\lambda_{1}, \lambda_{N}\right] \subset \mathbf{R}$ is the image of $P$ under $f$. Choose an intermediate point $\mu_{i}$ in each open interval $\left(\lambda_{i}, \lambda_{i+1}\right)$. Denote by $L_{i}$ (resp. $M_{i}$ ) the poset of proper faces of the polytope $f^{-1}\left(\lambda_{i}\right)$ (resp. $\left.f^{-1}\left(\mu_{i}\right)\right)$. When a value $\lambda$ moves from $\mu_{i}$ to $\lambda_{i}$, each face of $f^{-1}(\lambda)$ contracts to some face of $f^{-1}\left(\lambda_{i}\right)$ of possibly smaller dimension. This defines a surjective morphism of 
posets $\alpha_{i}: M_{i} \rightarrow L_{i}$. Similarly, we have the degeneration in the other direction, defining a morphism $\beta_{i}: M_{i} \rightarrow L_{i+1}$ (see also Lemma 2.2).

Lemma 1.3. The poset $\omega\left(P, s_{f}, t_{f}\right)$ is isomorphic to the inverse limit of the diagram

$$
M_{1} \stackrel{\beta_{1}}{\longrightarrow} L_{2} \stackrel{\alpha_{2}}{\longleftarrow} M_{2} \stackrel{\beta_{2}}{\longrightarrow} L_{3} \stackrel{\alpha_{3}}{\longleftarrow} M_{3} \stackrel{\beta_{4}}{\longrightarrow} \cdots \stackrel{\beta_{N-2}}{\longrightarrow} L_{N-1} \stackrel{\alpha_{N-1}}{\stackrel{1}{\longrightarrow}} M_{N-1} .
$$

Proof of Lemma 1.3. By definition, an element of this inverse limit is a sequence $\left(\Gamma_{1}, \Gamma_{2}, \ldots, \Gamma_{N-1}\right)$ of polytopes, where $\Gamma_{i} \in M_{i}$ is a face of $f^{-1}\left(\mu_{i}\right)$, such that $\beta_{i}\left(\Gamma_{i}\right)=\alpha_{i+1}\left(\Gamma_{i+1}\right)$ for $i=1,2, \ldots, N-2$. Since $f$ is generic, we have either $\operatorname{dim} \Gamma_{i}=\operatorname{dim} \beta_{i}\left(\Gamma_{i}\right)=\operatorname{dim} \alpha_{i+1}\left(\Gamma_{i+1}\right)=\operatorname{dim} \Gamma_{i+1} \quad$ (which means that $\Gamma_{i}$ and $\Gamma_{i+1}$ are hyperplane sections of the same face of $P$ ) or $\beta_{i}\left(\Gamma_{i}\right)=\alpha_{i+1}\left(\Gamma_{i+1}\right)$ is a point. In either case we get a unique cellular string in $P$, and each cellular string in $P$ is obtained in this way.

Lemma 1.4. The inverse image under $\alpha_{i}$ of any upper interval $\left\{\Phi \in L_{i}: \Phi \geq \Gamma\right\}$ in $L_{i}$ is a contractible subposet of $M_{i}$, and similarly for the map $\beta_{i}$.

Proof of Lemma 1.4. We view $f^{-1}\left(\lambda_{i}\right), f^{-1}\left(\mu_{i}\right)$, and their normal cones as polyhedra in the kernel of $f$. Let $\mathscr{N}_{\Gamma}$ be the normal cone of $f^{-1}\left(\lambda_{i}\right)$ at $\Gamma$. A face $\Xi$ of $f^{-1}\left(\mu_{i}\right)$ lies in $\alpha_{i}^{-1}\left(\left\{\Phi \in L_{i}: \Phi \geq \Gamma\right\}\right)$ if and only if the normal cone of $f^{-1}\left(\mu_{i}\right)$ at $\Xi$ is contained in $\mathscr{N}_{\Gamma}$. Moreover, these cones form a polyhedral subdivision of $\mathscr{N}_{\Gamma}$. Hence $\alpha_{i}^{-1}\left(\left\{\Phi \in L_{i}: \Phi \geq \Gamma\right\}\right)$ is contractible.

It follows from Lemma 1.4 and Quillen's Theorem A [9] that the maps of geometric realizations $\left|\alpha_{i}\right|:\left|M_{i}\right| \rightarrow\left|L_{i}\right|$ and $\left|\beta_{i}\right|:\left|M_{i}\right| \rightarrow\left|L_{i+1}\right|$ are homotopy equivalences and that all their fibers are contractible.

Proof of Theorem 1.2. Let $X_{i}$ denote the poset inverse limit of the left segment of our diagram up to $M_{i}$. Since the operations "inverse limit" and "geometric realization" commute $[6$, Chapter $3, \S 3]$, the geometric realization $\left|X_{i}\right|$ is the inverse limit of the corresponding diagram of topological spaces. Consider the natural projection $p_{i}:\left|X_{i}\right| \rightarrow\left|X_{i-1}\right|$. Each fiber of $p_{i}$ is contractible since it coincides with some fiber of $\left|\alpha_{i}\right|$. Hence $p_{i}$ is a homotopy equivalence by Quillen's Theorem A [9]. Using induction on $i$, we conclude that $\left|X_{N-1}\right|=|\omega|$ is homotopy equivalent to $\left|X_{1}\right|$. However, $\left|X_{1}\right|$ is the boundary of the $(n-1)$ polytope $f^{-1}\left(\mu_{1}\right)$ and hence homeomorphic to $S^{n-2}$.

We note that Björner [5] has given another proof of Theorem 1.2 in the case where $P$ is a zonotope (i.e., a Minkowski sum of line segments).

\section{COHERENT STRINGS AND THE MONOTONE PATH POLYTOPE}

We now sharpen Theorem 1.2 by identifying a subcomplex $\omega_{\text {coh }}$ of $\omega=$ $\omega\left(P, s_{f}, t_{f}\right)$ which is homeomorphic to $S^{n-2}$. To this end we introduce a larger space $\Omega$ homotopy equivalent to $\omega$, and we describe an explicit retraction from $\Omega$ onto $\omega_{\text {coh }}$.

Let $\Gamma$ denote the subdivision of the open segment $\operatorname{int}(Q)=\left(\lambda_{1}, \lambda_{N}\right)$ into open segments $\left(\lambda_{i}, \lambda_{i+1}\right)$ and singletons $\left\{\lambda_{j}\right\}, 1<j<N$. We call $\Gamma$ the chamber complex of $(P, Q)$. A face bundle is a map $\Delta$ which assigns to each $\sigma \in \Gamma$ a proper face $\Delta(\sigma)$ of $f^{-1}(\sigma)$. We usually identify $\Delta$ with a subcomplex of the boundary complex $\partial P$, whence the set $\mathscr{F}(P)$ of all face bundles is finite 
and partially ordered by inclusion. Every point $\psi=\left(\psi_{\sigma}\right)_{\sigma \in \Gamma} \in\left(S^{n-2}\right)^{\Gamma}$ defines a face bundle $\Delta_{\psi}$ via $\Delta_{\psi}(\sigma):=f^{-1}(\sigma)^{\psi_{\sigma}}$ (this denotes the extreme face of $f^{-1}(\sigma)$ in direction $\left.\psi_{\sigma}\right)$. Thus $\mathscr{F}(P)$ is the face poset of a piecewise-linear subdivision of the product of spheres $\left(S^{n-2}\right)^{\Gamma}$.

We define the monotone path complex $\mathscr{M}=\mathscr{M}_{f}(P)$ to be the subcomplex of $\mathscr{F}(P)$ consisting of all cells which are contained in

$$
\Omega:=\left\{\psi \in\left(S^{n-2}\right)^{\Gamma} \mid f^{-1}(\tau)^{\psi_{\tau}}=f^{-1}(\tau)^{\psi_{\sigma}} \text { whenever } \tau \subset \bar{\sigma}\right\} \text {. }
$$

Proposition 2.1. The monotone path complex $\mathscr{M}$ equals the cellular string complex $\omega$.

Proposition 2.1 is a consequence of Lemma 1.3 and the following Lemma 2.2 whose elementary proof we omit.

Lemma 2.2. For any $\psi \in S^{n-2}$, we have $\beta_{i-1}\left(f^{-1}\left(\mu_{i-1}\right)^{\psi}\right)=f^{-1}\left(\lambda_{i}\right)^{\psi}=$ $\alpha_{i}\left(f^{-1}\left(\mu_{i}\right)^{\psi}\right)$.

Using Proposition 2.1 and a covering argument, it can be shown that the cellular string complex $\omega$ is homotopy equivalent to the space $\Omega$. We now consider its diagonal

$$
\Omega_{\text {coh }}:=\left\{\psi \in\left(S^{n-2}\right)^{\Gamma} \mid \psi_{\sigma}=\psi_{\tau} \text { for all cells } \tau, \sigma \text { in } \Gamma\right\}
$$

which is clearly homeomorphic to the $(n-2)$-sphere. The following result yields an alternative proof of Theorem 1.2.

Theorem 2.3. The inclusion of topological spaces $\Omega_{\mathrm{coh}} \subset \Omega$ is a homotopy equivalence.

Proof. Let $\sigma_{1}<\sigma_{2}<\cdots<\sigma_{2 N-1}$ be the natural ordering of all cells of the chamber complex $\Gamma$ (thus $\sigma_{2 j-1}$ is a 1 -cell and $\sigma_{2 j}$ is a 0 -cell). We abbreviate $\psi_{i}:=\psi_{\sigma_{i}} \in S^{n-2}$ and consider the intermediate spaces

$$
\Omega_{i}:=\left\{\psi \in \Omega \mid \psi_{i}=\psi_{i+1}=\cdots=\psi_{2 N-1}\right\} \text { for } i=1,2, \ldots, 2 N-1 .
$$

Since $\Omega_{\text {coh }}=\Omega_{1} \subseteq \Omega_{2} \subseteq \cdots \subseteq \Omega_{2 N-1}=\Omega$, it suffices to show that the inclusion $\Omega_{i} \subseteq \Omega_{i+1}$ is a homotopy equivalence. We will prove this for the case $i=2 j-1$ (so $\sigma_{i}$ is a 1 -cell); the case $i=2 j$ is analogous.

Let $\psi \in \Omega_{i+1}$. The local coherence condition in (1) states that both $\psi_{i+1}$ and $\psi_{i}$ are support vectors for the same face of the fiber over the point $\sigma_{i+1}$. This implies $\psi_{i} \neq-\psi_{i+1}$, and thus the convex combinations

$$
\psi_{i}(\lambda)=\frac{\lambda \psi_{i}+(1-\lambda) \psi_{i+1}}{\left\|\lambda \psi_{i}+(1-\lambda) \psi_{i+1}\right\|} \quad \text { for } \lambda \in[0,1]
$$

are well-defined vectors on the unit sphere $S^{n-2}$. Now check that the point

$$
\psi(\lambda):=\left(\psi_{1}, \psi_{2}, \ldots, \psi_{i}, \psi_{i}(\lambda), \ldots, \psi_{i}(\lambda)\right) \in\left(S^{n-2}\right)^{\Gamma}
$$

lies in $\Omega$ for all $\lambda \in[0,1]$. This is clearly the case for $\lambda=0$, since $\psi(0)=\psi \in$ $\Omega_{i+1}$. On the other hand, the local coherence condition in (1) is preserved at each vertex $\sigma_{2 l}$ as the parameter $\lambda$ increases from 0 to 1 . Therefore, the map $\psi \mapsto \psi(1)$ provides the desired explicit retraction from $\Omega_{i+1}$ onto $\Omega_{i}$.

We define $\omega_{\text {coh }}$ to be the subposet of $\omega$ consisting of all face bundles (or cellular strings) $\Delta_{\psi}$ with $\psi \in \Omega_{\text {coh }}$. Such face bundles (or cellular strings) $\Delta_{\psi}$ 
are called coherent. It was shown in [3] that the poset $\omega_{\text {coh }}$ of coherent face bundles is isomorphic to the face poset of the $(n-1)$-dimensional monotone path polytope $\Sigma_{f}(P)$, which is defined as the Minkowski sum $\Sigma_{f}(P)=\sum_{\sigma \in \Gamma} f^{-1}(\sigma)$. Thus $\omega_{\text {coh }}$ is a natural subcomplex of $\omega$ which is homeomorphic to the $(n-2)$ sphere.

\section{EXAMPLES AND THE GENERALIZED BAUES PROBLEM}

We first discuss the three classes of examples mentioned in the introduction.

Example 3.1. The standard double stippling on the simplex $\Delta^{n}$ is defined as follows. Faces of $\Delta^{n}$ are identified with ordered subsets $\sigma=\left\{\sigma_{0}<\cdots<\sigma_{k}\right\} \subset$ $\{0,1,2, \ldots, n\}$, and we set $s(\sigma)=\sigma_{0}, t(\sigma)=\sigma_{k}$. It was noticed in $[1,2]$ that the cellular string complex $\omega\left(\Delta^{n}, s, t\right)$ is isomorphic to the boundary complex of the cube $I^{n-1}$. This double stippling is realized geometrically by the linear functional $f: \Delta^{n} \rightarrow \mathbf{R}$ which takes the value $i$ at the $i$ th vertex. The monotone path polytope $\Sigma_{f}\left(\Delta^{n}\right)$ is combinatorially (but not affinely) isomorphic to $I^{n-1}$. In fact, $\Sigma_{f}\left(\Delta^{n}\right)$ is the Newton polytope of the discriminant of a univariate polynomial of degree $n$ [7]. Here all cellular strings are coherent [3].

Example 3.2. The vertices of the $n$-cube $I^{n}=\left\{\left(x_{1}, \ldots, x_{n}\right) \in \mathbf{R}^{n} \mid 0 \leq x_{i} \leq\right.$ $1\}$ correspond to subsets of $\{1,2, \ldots, n\}$, which are partially ordered by inclusion. The standard combinatorial double stippling $(s, t)$ associates to each face its unique minimal (resp. maximal) vertex with respect to this order. The complex of cellular strings $\omega\left(I^{n}, s, t\right)$ is isomorphic to the face lattice of the $(n-1)$-dimensional permutohedron $P_{n}$ (see $[3,10]$, and below). This double stippling is realized geometrically by the linear functional $f\left(x_{1}, \ldots, x_{n}\right)=$ $x_{1}+\cdots+x_{n}$. It was shown in [3] that all the cellular strings are coherent and the monotone path polytope $\Sigma_{f}\left(I^{n}\right)$ is linearly isomorphic to $P_{n}$.

Example 3.3. The permutohedron $P_{n}$ is defined as the convex hull in $\mathbf{R}^{n}$ of the points $(\sigma(1), \sigma(2), \ldots, \sigma(n))$ for all permutations $\sigma \in S_{n}$. The face lattice of $P_{n}$ is well known (cf. [2,10]); in particular, two permutations $\sigma, \tau \in S_{n}$ are connected by an edge of $P_{n}$ if and only if they differ by an adjacent transposition (i.e., $\sigma=\tau \circ(i, i+1))$. The standard combinatorial double stippling of $P_{n}$ associates to each face $\Gamma \subset P_{n}$ its unique minimal vertex $s(\Gamma)$ (resp. maximal vertex $t(\Gamma))$ with respect to the weak Bruhat order. Recall that the weak Bruhat order on the symmetric group $S_{n}$ is defined as the transitive closure of the relation $\preceq$, where $\sigma \preceq \tau$ if $\sigma=\tau$ or $\sigma=\tau \circ(i, i+1)$ for some $i$ and the number of inversions in $\tau$ is larger than the number of inversions of $\sigma$.

The double stippling $(s, t)$ may be realized geometrically by the linear functional $f\left(x_{1}, \ldots, x_{n}\right)=\sum_{i=1}^{n} t_{i} x_{i}$, where $t_{1}<\cdots<t_{n}$ is any increasing sequence of real numbers. It follows from examples in [2, §III.7] that many of the cellular chains in $\omega\left(P_{n}, s, t\right)$ are noncoherent. The sphericity of the cellular string complex for $P_{n}$ was the original Baues's conjecture. Note that Baues's description in [2] is purely combinatorial, while our proof of the conjecture makes use of convex geometry.

We now discuss a natural generalization of the posets $\omega(P, f)$ for the case where the linear functional $f$ is replaced by a linear operator into $\mathbf{R}^{k}$. In the topological setting discussed in the introduction this corresponds to the $k$-fold 
loop space $\Omega^{k}(X)$ regarded as the space of pointed maps of the $k$-sphere to $X$ rather than iteratively as $\Omega\left(\Omega^{k-1}(X)\right)$.

Consider a convex polytope $P:=\operatorname{conv}\left(\mathscr{A}_{P}\right)$, where $\mathscr{A}_{P}=\left\{p_{1}, p_{2}, \ldots, p_{m}\right\}$ $\subset \mathbf{R}^{n}$, and let $\pi: \mathbf{R}^{n} \rightarrow \mathbf{R}^{d}$ be an affine map with $\pi\left(p_{1}\right)=q_{1}, \ldots, \pi\left(p_{m}\right)=q_{m}$. We consider $\mathscr{A}_{Q}=\left\{q_{1}, q_{2}, \ldots, q_{m}\right\} \subset \mathbf{R}^{d}$ as an $m$-element multiset, and we define $Q:=\operatorname{conv}\left(\mathscr{A}_{Q}\right)$.

A polyhedral complex is a collection of convex polytopes having the property that the intersection of any two is a face of each and is itself in the collection. A polyhedral subdivision of $Q$ is a collection $\Pi$ of subsets of $\mathscr{A}_{Q}$ whose convex hulls form a polyhedral complex whose union equals $Q$. A polyhedral subdivision $\Pi$ of $Q$ is said to be induced by $\pi$ from $P$ if

(1) each cell $\sigma \in \Pi$ is of the form $\pi\left(F_{\sigma}\right)$, where $F_{\sigma}$ is the intersection of $\mathscr{A}_{P}$ with a supporting hyperplane of $P$, and

(2) for each $\sigma, \sigma^{\prime} \in \Pi, F_{\sigma \cap \sigma^{\prime}}=F_{\sigma} \cap F_{\sigma^{\prime}}$.

Note that $\sigma=\pi\left(F_{\sigma}\right)$ uniquely specifies the set $F_{\sigma}$. Also note that $\operatorname{dim} \sigma \leq$ $\operatorname{dim} F_{\sigma}$, where by the dimension of a set we mean the dimension of its affine span. An induced subdivision $\Pi$ is called tight if $\operatorname{dim} \sigma=\operatorname{dim} F_{\sigma}$ for each $\sigma \in \Pi$. Such subdivisions are just polyhedral sections of the projections $\pi$ : $P \rightarrow Q$, i.e., they are subcomplexes in $P$ which project onto $Q$ bijectively. If $P$ is a simplex, then the tight induced subdivisions of $Q$ are precisely the triangulations of $Q$ with vertices in $\mathscr{A}_{Q}$.

A polyhedral subdivision $\Pi$ of $Q$ is said to be proper if $\Pi \neq\left\{\mathscr{A}_{Q}\right\}$. Let $\mathscr{S}(P, Q)$ denote the set of all proper induced polyhedral subdivisions of $Q$. This set is partially ordered by inclusion, so we view $\mathscr{S}(P, Q)$ as a subposet of the partition lattice on $A_{Q}$.

It can be viewed as a combinatorial model of the space of all continuous sections of the projection $\pi: P \rightarrow Q$ lying in the boundary of $Q$. In the case where $\operatorname{dim} Q=1$, the poset $\mathscr{S}(P, Q)$ is the poset $\omega$ of cellular strings. In view of Theorem 1.2, it is natural to ask the following question.

Generalized Baues Problem. Is the poset $\mathscr{S}(P, Q)$ homotopy equivalent to a sphere of dimension $\operatorname{dim} P-\operatorname{dim} Q-1$ ?

As in $\S 2$, there is a natural candidate for a spherical subcomplex of $\mathscr{S}(P, Q)$. This is the boundary complex of the fiber polytope $\Sigma(P, Q)$ which was introduced in [3] as the Minkowski integral of all fibers of the projection $\pi$. When $\operatorname{dim} Q=1$, this is the monotone path polytope $\Sigma_{f}(P)$, where $f$ denotes the linear map from $P$ onto $Q$. As was shown in [3], the faces of $\Sigma(P, Q)$ correspond to $P$-coherent polyhedral subdivisions of $Q$. These can be defined analogously to $\S 2$. Thus the set of coherent polyhedral subdivisions forms a spherical subposet in $\mathscr{S}(P, Q)$.

Thus the essence of the problem is to compare two ways of averaging the fibers: one combinatorial, by means of the inverse limit (cf. Lemma 1.3) and the other geometric, by means of Minkowski summation of polytopes.

In general, it is already unknown whether the complex $\mathscr{S}(P, Q)$ is connected. A partial positive answer to the Generalized Baues Problem, this connectedness, would imply that any two triangulations of a simplicial polytope $Q$ can be joined by a sequence of bistellar moves [4] involving its vertices (consider the case where $P$ is a simplex). Positive results have been obtained in [11] for 
the case where $P$ is a regular $n$-cube for specific choices of $Q$, including those where $Q$ has dimension at most 2 or at least $n-3$. We can also obtain a positive answer in the case where $P$ is a simplex and $\operatorname{dim} Q=2$.

\section{REFERENCES}

1. J. F. Adams, On the cobar construction, Proc. Nat. Acad. Sci. U.S.A. 42 (1956), 409-412.

2. H. J. Baues, Geometry of loop spaces and the cobar construction, Mem. Amer. Math. Soc., vol. 25, Amer. Math. Soc., Providence, RI, 1980.

3. L. J. Billera and B. Sturmfels, Fiber polytopes, Ann. of Math. (2) 135 (1992), 527-549.

4. L. J. Billera, P. Filliman, and B. Sturmfels, Constructions and complexity of secondary polytopes, Adv. Math. 83 (1990), 155-179.

5. A. Björner, Essential chains and homotopy type of posets, Proc. Amer. Math. Soc. 116 (1992), 1179-1181.

6. P. Gabriel and M. Zisman, Calculus of functions and homotopy theory, Ergebnisse der Math., bd. 35, Springer, Berlin, 1967.

7. I. M. Gelfand, M. M. Kapranov, and A. V. Zelevinsky, Newton polytopes of the classical discriminant and resultant, Adv. Math. 84 (1990), 237-254.

8. R. J. Milgram, Iterated loop spaces, Ann. of Math. (2) 84 (1966), 386-403.

9. D. Quillen, Higher algebraic $K$-theory: I, Higher K-Theories (H. Bass, ed.), Lecture Notes in Math., vol. 341, Springer, New York, 1973, pp. 77-139.

10. R. Rado, An inequality, J. London Math. Soc. (2) 27 (1952), 1-6.

11. B. Sturmfels and G. Ziegler, Extension spaces of oriented matroids, Discrete Comput. Geom. 10 (1993), 23-45.

(L. J. Billera and B. Sturmfels) Department of Mathematics, Cornell University, IthaCa, NEW YORK 14853

E-mail address: billera@math.cornell.edu

E-mail address: bernd@math.cornell.edu

(M. M. Kapranov) Department of Mathematics, Northwestern University, Evanston, ILLINOIS 60208

E-mail address: kapranov@chov.math.nwu.edu 